\title{
New studies reveal how autism might alter synapse formation, pruning
}

\section{BY ANGIE VOYLES ASKHAM}

12 NOVEMBER 2021

Two new unpublished studies presented virtually at the 2021 Society for Neuroscience annual meeting offer insights into synapse development: One maps the trajectories of synapse formation across nine species, and the other characterizes the earliest synapses to arise in the human brain.

The findings could help researchers better understand how developmental changes may alter synaptic function and contribute to autism.

"In order to understand whether something is deviated from neurotypical, you actually have to know what neurotypical is," says Sam Wang, professor of neuroscience at Princeton University and principal investigator on one of the new studies.

Across species, early brain development is defined by a period of exuberant synapse formation, followed by one in which any unnecessary connections are pruned. Disruption to either process may explain some of the atypical development seen in autism, but much about synaptic development remains unknown.

For example, when Wang and his colleagues began sifting through the literature to figure out when in development cortical synapses are most abundant and whether that timing shows shared patterns across species, they couldn't find any studies that had charted the full developmental trajectory from birth to adulthood, says Henk-Jan Boele, a postdoctoral researcher in Wang's lab who presented the work. So they decided to plot that course themselves, for as many species of mammals as they could find data for.

\section{Laws of maturation:}

Wang and Boele whittled down their initial literature search to 130 studies that logged synapse 


\section{Spectrum | Autism Research News}

https://www.spectrumnews.org

density counts at several time points for a variety of mammals, including humans, marmosets, cats, rabbits and mice. From those studies, the team extracted more than 16,000 unique data points to analyze and plot by species.

Each species analyzed differs in the number of days it takes for the cortex to reach peak synaptic density, the researchers found, but the time frame scales with the length of the animal's gestational period. And for all of the species studied, synaptic density peaked around one gestational period after birth.

"We were not expecting this," Wang says. "It's like there's this universal scaling law of cortical maturation."

Other aspects of development did not hold up across species: In human brains, cortical synapses mature first in the occipital lobe at the back of the brain and then steadily progresses frontward, whereas macaque monkeys' cortical areas seemed to mature simultaneously across the brain peaking in synaptic density at the same time and then steadily decreasing as connections are pruned.

Overlaying data points from autistic people or autism model mice onto the curves suggested that the condition is linked to a shallower decrease in synaptic density following the initial peak, which could be explained by inadequate pruning.

Wang and Boele next plan to explore how early postnatal changes to the cerebellum, a brain area that some researchers suggest facilitates cortical maturation and contributes to autism, shape these trajectories.

Growth curve: Most mammals studied reach peak synaptic density about one gestational period after birth.

\section{Early signals:}

The other new study explored how synapses form in the first place.

The researchers injected a form of rabies virus into a single neuron in slices of postmortem fetal brain tissue. The virus transmits to cells that synapse onto the injected cell and causes them to fluoresce green, revealing their organization.

Most cells in these synaptic snapshots were excitatory, the team found, and had immature electrical properties. The neurons also exhibited low levels of spontaneous calcium signaling, 


\section{Spectrum | Autism Research News}

https://www.spectrumnews.org

which is thought to be necessary for the formation of new synapses during development.

"Synchronous activity may help [the cells] to recognize each other and form connections with each other," says study investigator Li Zhou, a postdoctoral researcher in Arnold Kriegstein's lab at the University of California, San Francisco, who presented the work.

Adding a serotonin receptor antagonist to cultured prenatal brain tissue dampened this spontaneous activity and, in a separate experiment, decreased the number of synapses in the tissue, the team found.

That means that serotonin may manipulate neuronal activity at this stage of development, Zhou says.

Moving forward, Zhou plans to examine how other types of receptors affect this calcium signaling. He also plans to study these developmental mechanisms in organoids, which can be more easily manipulated in the lab than fetal brain tissue.

Read more reports from the 2021 Society for Neuroscience virtual annual meeting.

Cite this article: https://doi.org/10.53053/WMPH7503 